\title{
Analysis of Structure of Efficient Encoding Approach of Turbo Decoder
}

\author{
Khushbu Shah \\ Parul Institute of Engineering \& Technology \\ Limda, Gujarat
}

\author{
Brijesh Vala \\ Parul Institute of Engineering \& Technology \\ Limda, Gujarat
}

\begin{abstract}
To deal with $\mathrm{n}$ numbers of user simultaneously and error free communication with maximum utilization of limited spectrum, BER(bit error rate) improvement is an open challenge for communication engineers. In this paper work is an attempt to implement such an error control code Turbo code in which BER improve by using various efficient encoding and decoding designs. Turbo code provides modest decoding complexity for large block length and better bit error rate as compared to other code. According to [7] RSC encoder provides minimum error probability is implemented. For testing AWGN wireless channel is used. Recursive structure along with BPSK modulation is used. The decoding algorithm such as Viterbi algoritm,MAP(maximum a posterior),BCJR (bahl, cocke, jelinek and raviv)can be used .
\end{abstract}

\section{Keywords}

BER (bit error rate), RSC (Recursive systematic codes)

\section{INTRODUCTION}

In Previous years requirement of user is very small like KB, MB. Day to day user's requirement is increasing in terms of $\mathrm{GB}, \mathrm{TB}$, etc. Due to that reason channel capacity is also increased which introduce noise in channel which affect the BER (bit error rate) performance. So that transmitted information may not be reliable. Presently Turbo code is in usage for this presently. So, the basic purpose of my dissertation is to improve BER and need technology to achieve available spectrum efficiency.

Turbo codes were introduced in 1993 at the International conference on communication (ICC) by Berrou, Glavieux and Thitimajshima in their paper "Near shennon's limit error correction coding and decoding-Turbo codes". In this paper, they mention a BER performance of $10^{-5}$ at an $\mathrm{E}_{\mathrm{b}} / \mathrm{N} 00.7 \mathrm{~dB}$ using only a $1 / 2$ rate code, generating tremendous interest in the field. Turbo codes performs well in the low SNR scenario. At high SNRs, some of the traditional codes like the reedSolomon code can have comparable or better performance than turbo codes. Even though Turbo codes can be considered as a block codes, they do not exactly work like block codes. Turbo codes are actually a quasi-mix between Block and Convolutional codes [1].

A convolutional code is a type of error-correcting code in which each m-bit information symbol (each m-bit string) to be encoded is transformed into an n-bit symbol, where $\mathrm{m} / \mathrm{n}$ is the code rate $(n \geq m)$, and the transformation is a function of the last $\mathrm{k}$ information symbols, where $\mathrm{k}$ is the constraint length of the code. Convolutional codes are commonly specified by three parameters; (n, k,m) [1].

$\mathrm{n}=$ number of output bits

$\mathrm{k}=$ number of input bits $\mathrm{m}=$ number of memory registers

A block code is any member of the large and important family of error-correcting codes that encode data in blocks. The term block code may also refer to any error correcting code that acts on a block of $\mathrm{k}$ bits input data to produce $\mathrm{n}$ bits of output data $(\mathrm{n}, \mathrm{k})$. The rate of the code is $\mathrm{k} / \mathrm{n}$. Each message word is associated with one and only one codeword. The total number of codewords in a code equals that of message words, $2 \mathrm{k}$. It follows from the property of subspace that linear block codes have the following two important properties:

1. The sum of any two codewords in $\mathrm{C}$ is another codeword in $\mathrm{C}$ :

$\mathrm{c}_{\mathrm{i}}+\mathrm{c}_{\mathrm{j}}=\mathrm{c}_{\mathrm{k}}$

Where $c_{i}, c_{j}$ and $c_{k} € C$.

2. There exists a set of $\mathrm{k}$ codewords in $\mathrm{C}$ which are linearly independent such that every codeword in $\mathrm{C}$ is a linear combination of the $\mathrm{k}$ codewords:

$\mathrm{c}=\mathrm{m}_{0} \mathrm{~g}_{0}+\mathrm{m}_{1} \mathrm{~g}_{1}+\ldots \ldots+\mathrm{m}_{\mathrm{k}-1} \mathrm{~g}_{\mathrm{k}-1}$

Where $\mathrm{g}_{0}, \mathrm{~g}_{1}, \mathrm{~g}_{2}, \ldots . . \mathrm{g}_{\mathrm{k}-1}$ are the $\mathrm{k}$ linearly independent codewords, and $\mathrm{m} 0, \mathrm{~m} 1, \ldots \ldots, \mathrm{mk}-1$ are some scalars. Linearly independent it means that $\mathrm{m}_{0} \mathrm{~g}_{0}+\mathrm{m}_{1} \mathrm{~g}_{1}+\ldots \ldots+\mathrm{m}_{\mathrm{k}-1} \mathrm{~g}_{\mathrm{k}-1} \neq 0$ unless $\mathrm{m}_{0}$ $, \mathrm{m}_{1}, \ldots \ldots, \mathrm{mk}-1=0[4]$.

\section{TURBO ENCODER}

This section contains details about turbo encoder. Figure: 1 shows basic block diagram of communication system. The input signal is given to information source and passes to source encoder. The main purpose of source encoder is to transforms output source to binary digit i.e. information sequence $(u)$ then it passes to channel encoder it will transforms information sequence to discrete encoded sequence i.e. codeword (v). Instead of channel encoder turbo encoder is going to used which contains two RSC (Recursive Systematic Convolutional) encoders with an inter-leaver separating them. Figure: 2 show turbo encoder structure. Those discrete symbols are not suitable for transmission over physical channel. So, that the modulator transforms each output symbols of channel encoder to wave form that is enters in the channel and corrupted by noise. The demodulator processes each wave form and may be produced discrete output. Channel encoder transforms this received sequence to estimated sequence. And finally source encoder transforms estimated sequence to estimated output. 


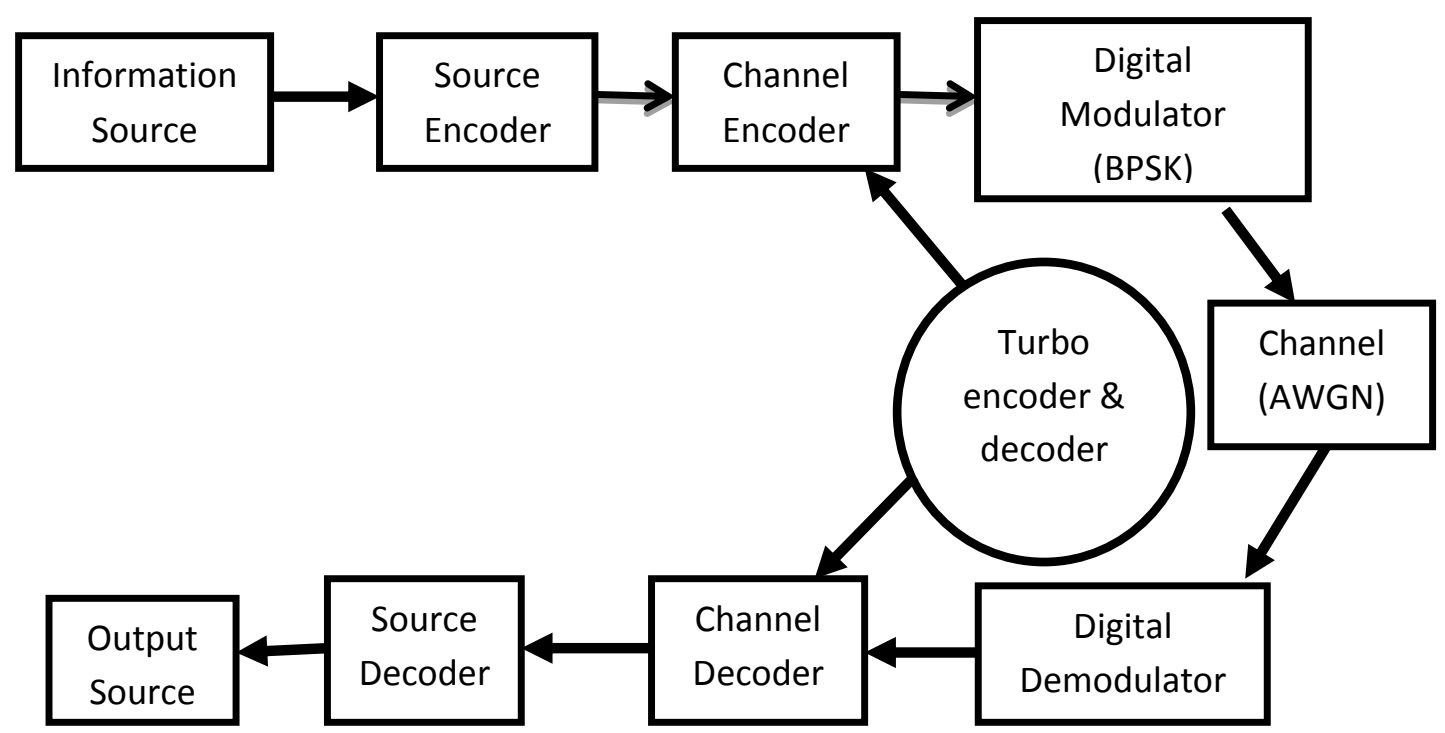

Figure 1: Role of Turbo code in communication system

\section{RSC (RECURSIVE SYSTEMATIC CONVOLUTIONAL) ENCODER}

This section contains brief concept of Recursive Systematic Convolutional encoders. It consists of two RSC (Recursive Systematic Convolutional) with an inter-leaver separating them. The block diagram for turbo encoder is shown in figure:
2. It is comprised of two transfer functions which represent the systematic components of RSC encoders and an interleaver. The input symbols are simply permuted in a random fashion and forwarded to the second RSC encoder [5].

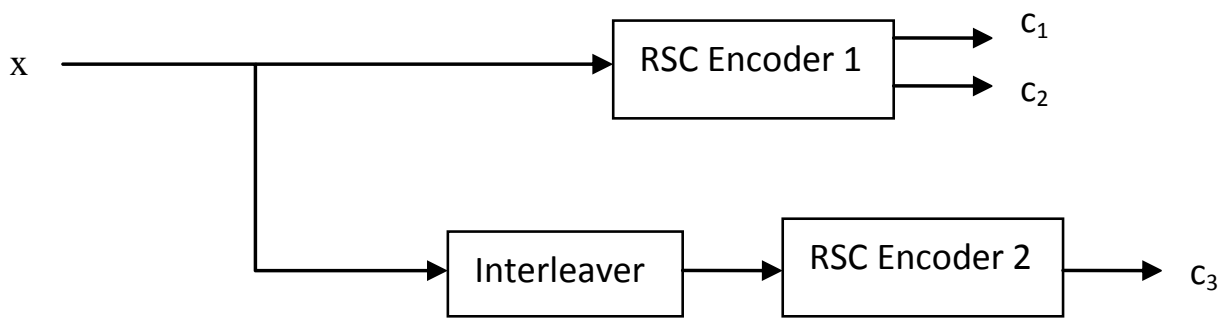

Figure 2: Recursive Systematic Convolutional encoder

Coding techniques such as convolutional codes are suitable for channels with random errors like binary symmetric channel or AWGN channel. But there are many channels where errors occur continuously. A burst of errors produces large errors in code words. So, there appears a need of strong correction capability. In order deal with these bursty channels, inter-leaver is introduced. Inter-leaver works by taking an input sequence and permuting it randomly or according to a prescribed method [5].

The function of interleaver is that suppose codeword (c1) produced by RSC encoder1 is low weight codeword means hamming distance (no of non-zero codewords) not much. Codeword produced by RSC encoder 2 is most likely not necessarily produced will be high weight codeword (c3).this is ideal for the code because high weight codewords result in better decoder performance. Although the encoder determine the capability for the error correction and decoder that determines the actual performance [1].

\section{CHANNEL}

Channel as AWGN (additive white Gaussian noise) 'Additive' because it is added to any noise that might be intrinsic to the information system. 'White' refers to idea that it has uniform power across the frequency band for the information system.
It is an analogy to the color white which has uniform radiations at all occurrences in the observable spectrum. 'Gaussian' because it has a usual distribution in the time domain with an average time domain value of zero. The channel capacity for the AWGN channel is given by:

$$
\mathrm{C}=1 / 2 \log (1+\mathrm{P} / \mathrm{N})
$$

Where, $\mathrm{P}$ represents the maximum channel power \& $\mathrm{N}$ represents Noise [6]

\section{IMPLEMENTATION}

The implementation was carried out in MATLAB programming Language. MATLAB (matrix laboratory) is a multi-paradigm numerical computing environment. It permits manipulations of matrix, functions plotting and data, creation of user interfaces and implementation of algorithm. MATLAB has user friendly and interactive environment which gives several in built functional facilities like Variables, Vectors/ Matrices, Structures, Functions, Classes, Graphics, GUI, Object oriented programming, Interfacing with other programming languages. 


\section{TURBO DECODER}

There are different decoding algorithms for turbo code such as Viterbi decoding, MAP (Maximum a posterior), SOVA(soft output Viterbi algorithm), etc. From the literature survey, Maximum A Posteriori algorithm, sometimes also called as BCJR(bahl, cocke, jelinek and raviv) algorithm, offerings an optimal decoding method for linear codes which minimizes the symbol error possibility. This is different from usually used Viterbi algorithm, In essence the Viterbi algorithm minimizes the probability of sequence (or word) error, which does not translate to minimizing the probability of individual bit (symbol) errors[1]. Below Table 1. Shows the complexity comparison of various decoding algorithm, where $\mathrm{M}$ is memory order.

Table 1. Complexity comparison of various decoding algorithm [8]

\begin{tabular}{|c|c|}
\hline Decoding Algorithm & Complexity \\
\hline Viterbi & $10 \cdot 2^{\mathrm{M}}+3$ \\
\hline SOVA & $17 \cdot 2^{\mathrm{M}}+4 \cdot(5 \mathrm{M})+4$ \\
\hline Log-MAP-algorithm & $48 \cdot 2^{\mathrm{M}}-13$ \\
\hline Max-Log-MAP-algorithm & $28 \cdot 2^{\mathrm{M}}-3$ \\
\hline
\end{tabular}

\section{CONCLUSION AND FUTURE SCOPE}

From the literature survey concluded that as no of users are increases, capacity of channel should be increase so there may be chance of increasing noise and it will affect BER of the channel. So, there must be need to provide reliable transmission of data by improving bit error rate for turbo code which is used in most real world applications. This can be implemented on hardware like DSP processor and FPGA to encode data efficiently and faster. Further complexity can be reduced by keeping systematic convolutional code stages.

\section{REFERENCES}

[1] "Information Theory and Coding" By-Ranjan Bose

[2] "Mathematical methods and algorithms" By- Todd K. Moon

[3] "Digital Communications Fundamentals and Applications" By- Bernard Sklar

[4] "A Practical Guide to Error-Control Coding Using MATLAB” By-Yuan Jiang

[5] Nabeel Arshad, Abdul Basit,"Implementation and Analysis of Turbo Codes Using MATLAB", Journal of Expert Systems (JES) 2013

[6] http://en.m.wikipedia.org/wiki/coding_thepy

[7] Nabeel Arshad, Abdul Basit," Implementation and Analysis of Turbo Codes Using MATLAB", Journal of Expert Systems (JES) 2013

[8] Ioannis A. Chatzigeorgiou, Miguel R. D. Rodrigues, Ian J. Wassell and Rolando Carrasco" A Comparison of
Convolutional and Turbo Coding Schemes For Broadband FWA Systems"

[9] L.Hadj Abderrahmane, M.Bacha and A.Mebrek" A New Optimised Interleaver Structure for Turbo Coding", 978-1-4799-3010-9/14/\$31.00 @2014 IEEE

[10] A. Al-Dweik,S. Le Goff, and B. Sharif ," A Hybrid Decoder for Block Turbo Codes", 0090-6778/09\$25.00 c 2009 IEEE

[11] Chair of Wireless Communications, Poznan University of Technology, Poznan, Poland,'Decoding of Serial Concatenated Convolutional Codes Using Log-MAP Delta Algorithm", 978-1-4577-1473-3/12/\$26.00 @2012 IEEE

[12] Ibrahim S. Raad, Mehmet Yakan "Implementation of a turbo codes test bed in the Simulink environment", 07803-9243-4/05/\$20.00 @2005 IEEE

[13] Pen-Yao Lu, Erl-Huei Lu, and Tso-Cho Chen,"An Efficient Hybrid Decoder for Block Turbo Codes",IEEE 2014

[14] Zhongfeng Wang and Keshab K. Parhi," High Performance, High Throughput Turbo/SOVA Decoder Design”, 0090-6778/03\$17.00 @ 2003 IEEE

[15] Punya THITIMAJSHMA "Recursive Systematic Convolutional Codes And Application To Parallel Concatenation", 0-7803-25091-5/95 US\$4.00 01995 IEEE 